\title{
INTERNATIONAL RESEARCH JOURNAL OF PHARMACY
}

\author{
www.irjponline.com
}

ISSN $2230-8407$

\section{Research Article \\ SCREENING AND EVALUATION OF BIOACTIVE COMPONENTS OF CENCHRUS CILIARIS L. BY GC-MS ANALYSIS}

Sunita Arora *, Ganesh Kumar, Sonam Meena

Department of Botany, Jai Narain Vyas University, Jodhpur, India

*Corresponding Author Email: jnvusunitarora@gmail.com

Article Received on: 19/05/17 Approved for publication: 19/06/17

DOI: $10.7897 / 2230-8407.08699$

\section{ABSTRACT}

Plants are rich source of secondary metabolites with interesting biological activities. Cenchrus ciliaris L. is an important forage plant belonging to grass family (Poaceae). The objective of the present investigation was to characterize possible bioactive phytochemical constituents from the whole plant of C. ciliaris L. using methanol by gas chromatography-mass spectrometry (GC-MS) analysis. The shade-dried plant powder was extracted with methanol using Soxhlet extractor and crude extract was subjected to GC-MS. The phytochemical constituents were investigated using Perkin-Elmer gas chromatography-mass spectrometry, while the mass spectra of the compounds found in the extract was matched with the National Institute of Standards and Technology (NIST) and Willey 8 library. Maximum \% area is found for Stigmasta-5, 22-Dien-3-ol, is present in maximum amount $(12.68 \%)$ with RT $=36.461 \mathrm{~min}$. in the methanolic root extract. 6,6-Dideutero-nonen-1-ol-3, is present in maximum amount $(31.92 \%)$ with $\mathrm{RT}=12.088 \mathrm{~min}$. in the methanolic stem extract and Pentadecanoic acid is present in maximum amount $(20.04 \%)$ with RT=16.328 min. in the leaf extract of Cenchrus ciliaris L. Green plants synthesize and preserve a variety of biochemical compounds, these products include flavonoids, phenols, saponins, terpenes, steroids and glycosides. C4 plants, specifically in warm conditions may show more potentialities to defend themselves. These compounds are commercially important and are used by pharmaceutical industries as well as the traditional practitioners for making herbal/synthetic drug formulations.

Keywords: Cenchrus ciliaris L., Gas Chromatography - Mass Spectroscopy, Secondary metabolites, Pharmaceutical, Antibiotics, Therapeutic drugs.

\section{INTRODUCTION}

Plants defend themselves from pathogens and other herbivore enemies by elaborating a variety of bioactive secondary metabolites that may have multiple molecular sites of action. Accordingly, exploitation of these useful plants has spread rapidly to safeguard increasing population from various pathogens and ailments. Many plants are good sources of antioxidants and other bioactive compounds containing phenolics, alkaloids, amino acids, ascorbic acid etc. Due to increasing demand, seeking therapeutic drugs from plants has grown tremendously. Such preparations contain various bioactive compounds of high therapeutic value and becoming popular in the area of medicine for their less expense and less side effects etc., compared to modern allopathic drugs. The traditional medicines in the last few decades emerged to have immense acknowledgements and it is estimated that $80 \%$ of community depend on traditional medicine for their primary healthcare ${ }^{1}$. More than $70 \%$ of India's 1.1 billion population still use non-allopathic systems of medicine ${ }^{2}$. Natural bioactive phytocompounds have been suggested as alternative sources for antibiotics. The chemical features of these constituents differ considerably among different species. This approach is alluring, in part, because they constitute a potential source of bioactive compounds that have been professed by the general public as comparatively safe and often act at multiple and novel target sites, thereby increasing the potential for resistance ${ }^{3}$. Extraction is the main step for the recovery and isolation of bioactive components from plant parts. The analysis and extraction of plant matrices play an important role in the development, modernization and quality control of herbal formulations ${ }^{4}$.The extraction of bioactive compounds from plants for therapeutic targets also needs active principle to be identified ${ }^{5}$.

GC-MS method can serve as an interesting tool for identification of active principles of herbs. It combines two analytical techniques to a single method of analyzing mixtures of chemical compounds. Gas chromatography separates the components of the mixture and mass spectroscopy analyzes each of the components separately ${ }^{6}$.

Cenchrus ciliaris, commonly known as Dhaman grass, is extremely variable, tufted and perennial species. This grass is gaining attention in various field of research as it is more competitive in high temperature, solar radiation and low moisture. It is most suitable and highly nutritive grass for environmental conditions of desert ${ }^{7}$.

\section{MATERIALS AND METHODS Collection of plant material}

Fresh, disease free plants of $C$. ciliaris were collected from natural habitats of Jodhpur (Rajasthan) during July to October. Further identification and authentication of the specimens was done from Botanical survey of India, Jodhpur (Raj.) The samples were washed thoroughly in running tap water to remove soil particles and other adhered debris and finally washed with sterile distilled water. The vegetative plant was dried under shade at room temperature and powdered using an electric grinder. After sieving the powder was transferred to airtight polyethylene zipper bags. 


\section{Preparation of plant extracts}

The powdered components (5 g) were successively extracted with methanol. Crude extracts of diff. plant parts were prepared with methanol by using hot extraction method $^{8}$ in soxhlet assembly. The extracts were stored at $-4{ }^{\circ} \mathrm{C}$ for further use Repeated extraction was done if needed with same solvent until a colorless extract was obtained. All the plant extracts were evaporated to dryness. The GC-MS analysis was performed at AIRF (Advanced Instrumentation Research Facility), Jawaharlal Nehru University, New Delhi, India. Identification of phytoconstituents was based on Willey 8 and NIST libraries attached to GC-MS instrument. Retention indices of extracted compounds were compared with stored components \& results were tabulated.

\section{RESULTS AND DISCUSSION}

Gas chromatography coupled with mass spectrometry is a commonly used technique for separating and identifying the components of complex volatile mixtures, it can be a valuable tool assisting in the separation and identification of isolated components. GC is known for its high resolution separation of structurally similar compounds. The use of electron ionization in mass spectrometry produces distinctive mass spectral fragmentation patterns enabling mass spectra for unknown to be searched against libraries ${ }^{9}$.

GC-MS chromatogram of the methanol extracts of root, stem, and leaf of $C$. ciliaris L. revealed 46, 46 and 66 peaks (Figure 13 ) indicating the presence of 42,44 and 61 bioactive compounds respectively. The GC-MS analysis of methanol extract of whole plant of C. ciliaris L. revealed the presence of 147 phytoconstituents that could contribute the medicinal quality of the plant. 26, $17 \& 35$ phytochemicals (table 1-3) were identified in root, stem \& leaf extract respectively with various biological activities. Structure and nature of common phytocompounds were identified in the methanolic extract of whole plant (Table 4). Mass spectrum of different compounds are shown (Figure 4-25).

Stigmasta-5, 22-Dien-3-OL ${ }^{10}$ is present in maximum amount (12.68\%), followed by Pentadecanoic acid (11.35\%), Stigmast5-en-3-ol, (3.beta.)- (8.50\%), Ergost-5-en-3-ol, (3.beta.,24r)$(7.08 \%)$ and Octadec-9-Enoic acid $(4.55 \%)$ in methanolic root extract. 6,6-Dideutero-nonen-1-ol-3 is present in maximum amount $(31.92 \%)$, followed by Pentadecanoic acid (11.50\%), Stigmasta-5,22-dien-3-ol $\quad(6.22 \%), \quad 9,12,15$-octadecatrienoic acid, $(\mathrm{z}, \mathrm{z}, \mathrm{z})-(5.43 \%)$, and 10,12 -Hexadecadien-1-ol $(4.49 \%)$ in methanolic stem extract and Pentadecanoic acid is present in maximum amount (20.04\%), followed by 9,12,15octadecatrienoic acid (z,z,z)- (10.67\%), Stigmasta-5,22-dien-3ol $(8.75 \%)$, Hexadecanoic acid, 2-hydroxy-1(hydroxymethyl)ethyl ester (3.96\%) and Stigmast-5-en-3-ol, (3.beta.)- (3.49\%), Ergost-5-en-3-ol, (3.beta.,24r)- (2.99\%) and Vitamin E $(2.82 \%)$ in methanolic leaf extract of C. ciliaris L. Phytosterols have been clinically proved to reduce blood cholesterol and scientific reports suggest that they posses antioxidant activity ${ }^{11}$. Beta-sitosterol is mainly known and used for its cholesterol lowering property ${ }^{12}$. Activities revealed by other valuable compounds have been tabulated. They are being used in a variety of ways in cure of various ailments \& in supplementing and supplanting human health and welfare ${ }^{13-18}$.

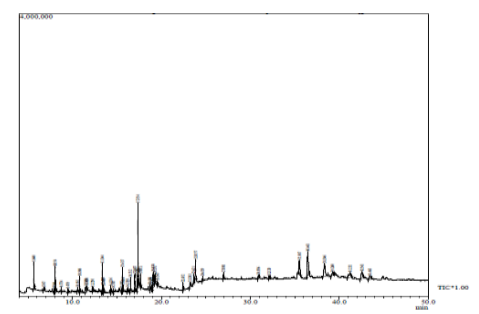

Figure 1: GC-MS Chromatogram of Cenchrus ciliaris L. methanolic root extract

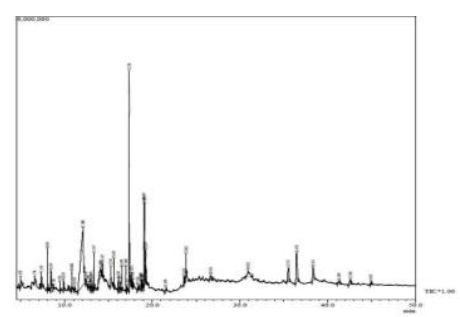

Figure 2: GC-MS Chromatogram of Cenchrus ciliaris L. methanolic stem extract

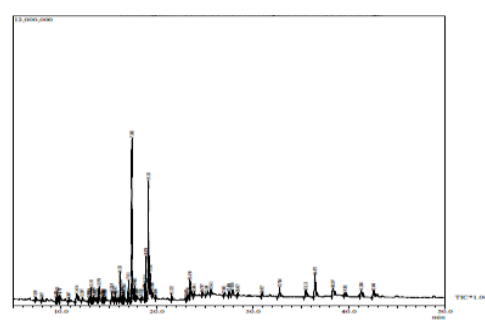

Figure 3: GC-MS Chromatogram of Cenchrus ciliaris L. methanolic leaf extract

Table 1: Bioactivity of compounds identified in the methanolic extract of root

\begin{tabular}{|c|c|c|c|c|c|}
\hline S. No. & RT (min.) & Compound & $\begin{array}{l}\text { Mol. } \\
\text { weight }\end{array}$ & $\begin{array}{l}\text { Mol. } \\
\text { formula }\end{array}$ & Bioactivities \\
\hline 1 & 6.767 & Hexanoic acid,2-Ethyl- & 144 & $\mathrm{C}_{8} \mathrm{H}_{16} \mathrm{O}_{2}$ & Plasticizer, Lubricants \\
\hline 2 & 7.944 & 1-Undecanol & 172 & $\mathrm{C}_{11} \mathrm{H}_{24} \mathrm{O}$ & Bactericidal \\
\hline 3 & 8.056 & Naphthalene & 128 & $\mathrm{C}_{10} \mathrm{H}_{28}$ & Antiseptic, Carcinogenic \\
\hline 4 & 9.479 & 2-Undecanone & 170 & $\mathrm{C}_{11} \mathrm{H}_{22} \mathrm{O}$ & $\begin{array}{l}\text { Natural non-toxic insect } \\
\text { repellant }\end{array}$ \\
\hline 5 & 10.806 & 1-Tetradecene & 196 & $\mathrm{C}_{14} \mathrm{H}_{28}$ & Anti tuberculosis \\
\hline 6 & 12.250 & Naphthalene, 2-bromo- & 207 & $\mathrm{C}_{10} \mathrm{H}_{7} \mathrm{Br}$ & Dye preparation \\
\hline 7 & 13.344 & 9-Eicosene, $(\mathrm{E})$ - & 280 & $\mathrm{C}_{20} \mathrm{H}_{40}$ & Antimicrobial, Cytotoxic \\
\hline 8 & 13.495 & $\begin{array}{l}\text { 1,2-Benzenedicarboxylic } \\
\text { acid, Diethyl ester }\end{array}$ & 222 & $\mathrm{C}_{12} \mathrm{H}_{14} \mathrm{O}_{4}$ & $\begin{array}{ll}\text { Cosmetics, } & \text { Insecticides, } \\
\text { Plasticizer } & \end{array}$ \\
\hline 9 & 14.316 & 8-Pentadecanone & 226 & $\mathrm{C}_{15} \mathrm{H}_{30} \mathrm{O}$ & $\begin{array}{l}\text { Hepatotoxic, Demyelination, } \\
\text { Conjunctivitis }\end{array}$ \\
\hline 10 & 14.585 & $\begin{array}{l}\text { Heptadecane, } \\
\text { tetramethyl- }\end{array}$ & 296 & $\mathrm{C}_{21} \mathrm{H}_{44}$ & Sex hormone in Algae \\
\hline 11 & 15.617 & n-Heptadecanol-1 & 256 & $\mathrm{C}_{17} \mathrm{H}_{36} \mathrm{O}$ & $\begin{array}{l}\text { Antiarthritis, In treatment of skin } \\
\text { diseases }\end{array}$ \\
\hline 12 & 15.686 & Tetradecane & 198 & $\mathrm{C}_{14} \mathrm{H}_{30}$ & $\begin{array}{ll}\text { Antifungal, } & \text { Antibacterial, } \\
\text { Nematicidal } & \\
\end{array}$ \\
\hline 13 & 16.198 & $\begin{array}{l}\text { 2-Pentadecanone, } \\
\text { Trimethyl- }\end{array}$ & 268 & $\mathrm{C}_{18} \mathrm{H}_{36} \mathrm{O}$ & Allelopathic, Antibacterial \\
\hline
\end{tabular}


Sunita Arora et al. Int. Res. J. Pharm. 2017, 8 (6)

\begin{tabular}{|c|c|c|c|c|c|}
\hline 14 & 17.007 & Octadecanoic acid, Methyl ester & 298 & $\mathrm{C}_{19} \mathrm{H}_{38} \mathrm{O}_{2}$ & $\begin{array}{lr}\text { Antifungal, } & \text { Antibacterial, } \\
\text { Antimicrobial, } & \text { Emulsifier, } \\
\text { Perfumery Industry } & \end{array}$ \\
\hline 15 & 17.267 & cis-13-Octadecenoic acid & 282 & $\mathrm{C}_{18} \mathrm{H}_{34} \mathrm{O}_{2}$ & $\begin{array}{l}\text { Therapeutic uses in medicine, } \\
\text { surgery }\end{array}$ \\
\hline 16 & 17.354 & Pentadecanoic acid & 242 & $\mathrm{C}_{15} \mathrm{H}_{30} \mathrm{O}_{2}$ & Lubricants, Adhesive agents \\
\hline 17 & 19.567 & n-Nonadecanol-1 & 284 & $\mathrm{C}_{19} \mathrm{H}_{40} \mathrm{O}$ & Antimicrobial, Cytotoxic \\
\hline 18 & 19.074 & Octadec-9-enoic acid & 282 & $\mathrm{C}_{18} \mathrm{H}_{34} \mathrm{O}_{2}$ & $\begin{array}{l}\text { Antihypertensive, Increases HDL } \\
\& \text { decrease LDL }\end{array}$ \\
\hline 19 & 19.271 & Octadecanoic acid & 284 & $\mathrm{C}_{18} \mathrm{H}_{36} \mathrm{O}_{2}$ & $\begin{array}{l}\text { Antifungal, Antitumor, } \\
\text { Antibacterial }\end{array}$ \\
\hline 20 & 22.432 & Octadecanal & 268 & $\mathrm{C}_{18} \mathrm{H}_{38} \mathrm{O}$ & Sex Pheromone \\
\hline 21 & 24.639 & 2-Hexadecyloxirane & 268 & $\mathrm{C}_{18} \mathrm{H}_{36} \mathrm{O}$ & $\begin{array}{lc}\text { Antibacterial, } & \text { Antimicrobial, } \\
\text { Antioxidant, } & \text { Antipyretic, } \\
\text { AntiInflammatory, Analgesic }\end{array}$ \\
\hline 22 & 30.936 & .gamma.-Tocopherol & 416 & $\mathrm{C}_{28} \mathrm{H}_{48} \mathrm{O}_{2}$ & $\begin{array}{l}\text { Antioxidant, Cardio protective, } \\
\text { Anticancer, AntiInflammatory }\end{array}$ \\
\hline 23 & 35.547 & Ergost-5-en-3-ol, (3.beta.,24r)- & 400 & $\mathrm{C}_{28} \mathrm{H}_{48} \mathrm{O}$ & $\begin{array}{lll}\text { Liver disease, Jaundice, } \\
\text { Arthrosclerosis }\end{array}$ \\
\hline 24 & 36.461 & Stigmasta-5,22-dien-3-ol & 412 & $\mathrm{C}_{29} \mathrm{H}_{48} \mathrm{O}$ & Synthetic Progesterone \\
\hline 25 & 38.346 & Stigmast-5-en-3-ol, (3.beta.)- & 414 & $\mathrm{C}_{29} \mathrm{H}_{50} \mathrm{O}$ & $\begin{array}{l}\text { AntiInflammatory, Antipyretic, } \\
\text { Anti ulcer, Antiarthritic }\end{array}$ \\
\hline 26 & 43.463 & $\begin{array}{l}\text { Androst-4-en-3-one, 17-hydroxy-, } \\
\text { (17.beta.)- }\end{array}$ & 288 & $\mathrm{C}_{19} \mathrm{H}_{28} \mathrm{O}_{2}$ & Regulation of spermatogenesis \\
\hline
\end{tabular}

Table 2: Bioactivity of compounds identified in the methanolic extract of stem

\begin{tabular}{|l|l|l|l|l|l|}
\hline $\begin{array}{l}\text { S. } \\
\text { No. }\end{array}$ & $\begin{array}{l}\text { RT } \\
(\text { min.) }\end{array}$ & Compound & $\begin{array}{l}\text { Mol. } \\
\text { Weight }\end{array}$ & $\begin{array}{l}\text { Mol. } \\
\text { formula }\end{array}$ & Bioactivities \\
\hline 1 & 7.339 & $\begin{array}{l}\text { 2,3-Dihydro-3,5- dihydro -6-methyll- } \\
\text { 4h-pyran-4-one }\end{array}$ & 144 & $\mathrm{C}_{6} \mathrm{H}_{8} \mathrm{O}_{4}$ & $\begin{array}{l}\text { Antimicrobial, } \\
\text { AntiInflammatory }\end{array}$ \\
\hline 2 & 8.050 & Napthalene & 204 & $\mathrm{C}_{10} \mathrm{H}_{8}$ & Antiseptic, Carcinogenic \\
\hline 3 & 8.429 & 2,3-Dihydro-benzofuran & 120 & $\mathrm{C}_{8} \mathrm{H}_{8} \mathrm{O}$ & $\begin{array}{l}\text { In treatment of Diabetic } \\
\text { Retinopathy and Arthritis }\end{array}$ \\
\hline 4 & 9.879 & 2-Methoxy-4-vinylphenol & 150 & $\mathrm{C}_{9} \mathrm{H}_{10} \mathrm{O}_{2}$ & Antibacterial \\
\hline 5 & 13.347 & 9-Eicosene & 280 & $\mathrm{C}_{20} \mathrm{H}_{40} \mathrm{O}$ & Antimicrobial, Cytotoxic \\
\hline 6 & 14.317 & 8-Pentadecanone & 226 & $\mathrm{C}_{15} \mathrm{H}_{30} \mathrm{O}$ & $\begin{array}{l}\text { Hepatotoxic, } \\
\text { Demyelination, } \\
\text { Conjunctivitis }\end{array}$ \\
\hline 7 & 9.470 & 2-Undecanone & 170 & $\mathrm{C}_{11} \mathrm{H}_{22} \mathrm{O}$ & Natural non-toxic insect repellent \\
\hline 8 & 15.258 & Tetradecanoic acid & 228 & $\mathrm{C}_{14} \mathrm{H}_{28} \mathrm{O}_{2}$ & $\begin{array}{l}\text { Antioxidant,Anticancer, } \\
\text { Hypocholesterolemic }\end{array}$ \\
\hline 9 & 16.509 & 8-Octadecanone & 268 & $\mathrm{C}_{18} \mathrm{H}_{36} \mathrm{O}$ & Antimicrobial \\
\hline 10 & 17.009 & $\begin{array}{l}\text { Hexadecanoic acid, } \\
\text { methyl ester }\end{array}$ & 270 & $\mathrm{C}_{17} \mathrm{H}_{34} \mathrm{O}_{2}$ & $\begin{array}{l}\text { Antioxidant, Antifungal, } \\
\text { Antimicrobial, Pesticide } \\
\text { Nematicide }\end{array}$ \\
\hline 11 & 17.675 & n-Nonadecanol-1 & 284 & $\mathrm{C}_{19} \mathrm{H}_{40} \mathrm{O}$ & Antimicrobial, Cytotoxic \\
\hline 12 & 18.688 & $\begin{array}{l}\text { 9,12-Octadecadienoic acid (z,z)-,methyl } \\
\text { ester }\end{array}$ & 294 & $\mathrm{C}_{19} \mathrm{H}_{34} \mathrm{O}_{2}$ & $\begin{array}{l}\text { Hepatoprotective, Anti-histaminic, } \\
\text { Antieczemic, Hypocholesterolemic }\end{array}$ \\
\hline 13 & 18.758 & $\begin{array}{l}\text { 9,12,15-Octadecatrienoic acid, methyl } \\
\text { ester, (z,z,z) }\end{array}$ & 292 & $\begin{array}{l}\text { AntiInflammatory, } \\
\text { Hypocholesterolemic, } \\
\text { preventive, Hepatoprotective }\end{array}$ \\
\hline 14 & 19.045 & $\begin{array}{l}\text { 10,12-Hexadecadien-1-ol } \\
\text { Cancer }\end{array}$ \\
\hline 16 & 21.509 & $\begin{array}{l}\text { 9-Octadecanoic acid (z)- } \\
\text { Octadecanal }\end{array}$ & 238 & $\mathrm{C}_{16} \mathrm{H}_{30} \mathrm{O}$ & Sex pheromone \\
\hline
\end{tabular}

Table 3: Bioactivity of phytochemical compounds identified in the methanol extract of leaf

\begin{tabular}{|l|l|l|l|l|l|}
\hline $\begin{array}{l}\text { S. } \\
\text { No. }\end{array}$ & $\begin{array}{l}\text { RT } \\
(\mathbf{m i n} .)\end{array}$ & Compound & $\begin{array}{l}\text { Mol. } \\
\text { Weight }\end{array}$ & $\begin{array}{l}\text { Mol. } \\
\text { formula }\end{array}$ & Bioactivities \\
\hline 1 & 7.339 & $\begin{array}{l}\text { 2,3-Dihydro-3,5-dihydroxy-6- } \\
\text { methyl-4h-pyran }\end{array}$ & 144 & $\mathrm{C}_{6} \mathrm{H}_{8} \mathrm{O}_{4}$ & $\begin{array}{l}\text { Antimicrobial, } \\
\text { AntiInflammatory }\end{array}$ \\
\hline 2 & 9.878 & 2-Methoxy-4-vinylphenol & 150 & $\mathrm{C}_{9} \mathrm{H}_{10} \mathrm{O}_{2}$ & Antibacterial \\
\hline 3 & 10.807 & 1-Tetradecene & 196 & $\mathrm{C}_{14} \mathrm{H}_{28}$ & Antituberculosis \\
\hline & & 283 & $\mathrm{C}_{10} \mathrm{H}_{13} \mathrm{~N}_{5} \mathrm{O}_{5}$ & $\begin{array}{l}\text { Cytotoxic against T cells lines, Anti viral } \\
\text { against Vero cells infected with HSV-1 }\end{array}$ \\
\hline 5 & 11.654 & Guanosine & 240 & $\mathrm{C}_{17} \mathrm{H}_{36}$ & Antioxidant \\
\hline 6 & 12.207 & Heptadecane & 200 & $\mathrm{C}_{12} \mathrm{H}_{24} \mathrm{O}_{2}$ & Antimicrobial \\
\hline
\end{tabular}


Sunita Arora et al. Int. Res. J. Pharm. 2017, 8 (6)

\begin{tabular}{|c|c|c|c|c|c|}
\hline 7 & 13.390 & Megastigmatrienone & 190 & $\mathrm{C}_{13} \mathrm{H}_{18} \mathrm{O}$ & Aroma \\
\hline 8 & 13.493 & $\begin{array}{l}\text { 1,2-Benzene dicarboxylic } \\
\text { acid, diethyl ester }\end{array}$ & 222 & $\mathrm{C}_{12} \mathrm{H}_{14} \mathrm{O}_{4}$ & Cosmetics, Insecticides, Plasticizer \\
\hline 9 & 14.364 & n- Tridecan-1-ol & 200 & $\mathrm{C}_{13} \mathrm{H}_{28} \mathrm{O}$ & Natural mosquito control agent \\
\hline 10 & 14.466 & 5-Octadecene, (E)- & 252 & $\mathrm{C}_{18} \mathrm{H}_{36}$ & Stronger sexual characters \\
\hline 11 & 14.589 & 2-Methyltetracosane & 352 & $\mathrm{C}_{25} \mathrm{H}_{52}$ & Free radical scavenging \\
\hline 12 & 15.260 & Tetradecanoic acid & 228 & $\mathrm{C}_{14} \mathrm{H}_{28} \mathrm{O}_{2}$ & $\begin{array}{l}\text { Antioxidant, Anticancer, } \\
\text { Hypocholesterolemic }\end{array}$ \\
\hline 13 & 16.133 & $\begin{array}{l}\text { 2,6,10-Trimethyl, 14-ethylene- } \\
\text { 14-pentadecne }\end{array}$ & 296 & $\mathrm{C}_{20} \mathrm{H}_{38}$ & Antiproliferative \\
\hline 14 & 16.204 & $\begin{array}{ll}\text { 2-Pentadecanone, } & 6,10,14- \\
\text { Trimethyl- }\end{array}$ & 268 & $\mathrm{C}_{18} \mathrm{H}_{36} \mathrm{O}$ & Allelopathic, Antibacterial \\
\hline 15 & 16.328 & Pentadecanoic acid & 242 & $\mathrm{C}_{15} \mathrm{H}_{30} \mathrm{O}_{2}$ & Lubricants, Adhesive agents \\
\hline 16 & 16.390 & $\begin{array}{l}\text { 2-Hexadecen-1-ol, } 3,7,11,15- \\
\text { tetramethyl-, }\left[\mathrm{r}-\left[\mathrm{r}^{*} \mathrm{r}^{*},-(\mathrm{e})\right]\right]\end{array}$ & 296 & $\mathrm{C}_{20} \mathrm{H}_{40} \mathrm{O}$ & Antimicrobial, Sedatives and anesthetics \\
\hline 17 & 16.520 & $\begin{array}{l}\text { 1,2-Benzenedicarboxylic } \\
\text { bis(2-Methylpropyl) Ester }\end{array}$ & 278 & $\mathrm{C}_{16} \mathrm{H}_{22} \mathrm{O}_{4}$ & Antimicrobial, Antifouling \\
\hline 18 & 17.011 & Hexadecanoic acid, methyl ester & 270 & $\mathrm{C}_{17} \mathrm{H}_{34} \mathrm{O}$ & $\begin{array}{l}\text { Antioxidant, Insecticide,hemolytic, Hypo - } \\
\text { cholesterolemic }\end{array}$ \\
\hline 19 & 17.183 & 13-Docosanoic acid & 338 & $\mathrm{C}_{22} \mathrm{H}_{42} \mathrm{O}_{2}$ & Surfactant, Precursor to Biodiesel fuel \\
\hline 20 & 17.268 & cis-13-Octadecenoic acid & 282 & $\mathrm{C}_{18} \mathrm{H}_{34} \mathrm{O}_{2}$ & Therapeutic uses in medicine, surgery \\
\hline 21 & 17.681 & Hexadecanoic acid, ethyl ester & 284 & $\mathrm{C}_{18} \mathrm{H}_{36} \mathrm{O}_{2}$ & $\begin{array}{l}\text { Antioxidant, hypocholesterolemic } \\
\text { Antiandrogenic, Hemolytic, 5-Alpha reductase } \\
\text { inhibitor }\end{array}$ \\
\hline 22 & 18.330 & Heptadecanoic acid & 270 & $\mathrm{C}_{17} \mathrm{H}_{34} \mathrm{O}_{2}$ & Antimicrobial \\
\hline 23 & 18.688 & $\begin{array}{l}\text { 9,12-Octadecadienoic acid (z,z,)-, } \\
\text { methyl ester }\end{array}$ & 294 & $\mathrm{C}_{19} \mathrm{H}_{34} \mathrm{O}_{2}$ & $\begin{array}{l}\text { Hepatoprotective, } \\
\text { Antieczemic, Hypocholesterolemic }\end{array}$ \\
\hline 24 & 18.758 & $\begin{array}{l}\text { 9,12,15-Octadecatrienoic acid, } \\
\text { methyl ester, }(\mathrm{z}, \mathrm{z}, \mathrm{z}) \text { - }\end{array}$ & 292 & $\mathrm{C}_{19} \mathrm{H}_{32} \mathrm{O}_{2}$ & $\begin{array}{l}\text { AntiInflammatory, Hypocholesterolemic, } \\
\text { Cancer preventive, Hepatoprotective }\end{array}$ \\
\hline 25 & 19.051 & 9,12 -Octadecadienoic acid $(\mathrm{z}, \mathrm{z},)^{-}$ & 280 & $\mathrm{C}_{18} \mathrm{H}_{32} \mathrm{O}_{2}$ & $\begin{array}{l}\text { AntiInflammatory, Antibacterial, Antiarthritic, } \\
\text { Hepatoprotectiv, } \\
\text { Anticoronary }\end{array}$ \\
\hline 26 & 19.133 & $\begin{array}{l}\begin{array}{l}\text { 9,12,15-Octadecatrienoic acid, } \\
(\mathrm{z}, \mathrm{z}, \mathrm{z})-\end{array} \\
\end{array}$ & 278 & $\mathrm{C}_{18} \mathrm{H}_{30} \mathrm{O}_{2}$ & Preventive against cardiovascular diseases \\
\hline 27 & 19.281 & Octadecanoic acid & 284 & $\mathrm{C}_{18} \mathrm{H}_{36} \mathrm{O}_{2}$ & Antifungal, Antitumor, Antibacterial \\
\hline 28 & 19.506 & $\begin{array}{l}\text { (E)-9-Octadecenoic acid ethyl } \\
\text { ester }\end{array}$ & 310 & $\mathrm{C}_{20} \mathrm{H}_{38} \mathrm{O}_{2}$ & Steroids and primer pheromone \\
\hline 29 & 19.846 & Phytol, acetate & 338 & $\mathrm{C}_{22} \mathrm{H}_{42} \mathrm{O}_{2}$ & flavor and fragrance \\
\hline 30 & 27.001 & Squalene & 410 & $\mathrm{C}_{30} \mathrm{H}_{50}$ & $\begin{array}{l}\text { Antioxidant, Anticancer Pesticide, Sunscreen, } \\
\text { Perfumery, Chemo preventive }\end{array}$ \\
\hline 31 & 30.927 & gamma.-Tocopherol & 416 & $\mathrm{C}_{28} \mathrm{H}_{48} \mathrm{O}_{2}$ & $\begin{array}{l}\text { Antioxidant, Cardio protective, Anticancer, } \\
\text { Anti-inflammatory }\end{array}$ \\
\hline 32 & 32.784 & Vitamin E & 430 & $\mathrm{C}_{29} \mathrm{H}_{50} \mathrm{O}_{2}$ & $\begin{array}{l}\text { Antiaging, Analgesic, antidiabetic, } \\
\text { Antidermatitic, Antileukemia, Anticancer, } \\
\text { Vasodilator, Hepatoprotective, } \\
\text { Hypocholesterolemic, } \\
\text { Antibronchitic, Anticoronary }\end{array}$ \\
\hline 33 & 35.513 & Ergost-5-en-3-ol, (3.beta.,24r)- & 400 & $\mathrm{C}_{28} \mathrm{H}_{48} \mathrm{O}$ & Liver disease, Jaundice, Arthrosclerosis \\
\hline 34 & 36.475 & Stigmasta-5,22-dien-3-ol & 412 & $\mathrm{C}_{29} \mathrm{H}_{48} \mathrm{O}$ & Synthetic Progesterone \\
\hline 35 & 38.337 & Stigmast-5-en-3-ol, (3.beta.)- & 414 & $\mathrm{C}_{29} \mathrm{H}_{50} \mathrm{O}$ & $\begin{array}{l}\text { Anti Inflammatory, Antipyretic, Anti ulcer, } \\
\text { Antiarthritic }\end{array}$ \\
\hline
\end{tabular}

Table 4: Structure and nature of compounds identified in the methanolic extract of whole plant

\begin{tabular}{|l|l|l|l|}
\hline S. No. & Compound & Nature & Structure \\
\hline 1 & Naphthalene & Polycyclic aromatic hydrocarbon & \\
\hline 2 & 2-Undecanone & Ketone & \\
\hline 3 & 9-Eicosene, (E)- & Alkene & \\
\hline 4 & 1-Tetradecene & Olefins & \\
\hline
\end{tabular}


Sunita Arora et al. Int. Res. J. Pharm. 2017, 8 (6)

\begin{tabular}{|c|c|c|c|}
\hline 5 & 8-Pentadecanone & Ketone & \\
\hline 6 & 2-Pentadecanone, 6,10,14-Trimethyl- & Sesquiterpenoids & $x-x^{-\cdots}$ \\
\hline 7 & cis-13-Octadecenoic acid & Elaidic acid & \\
\hline 8 & Pentadecanoic acid & Fatty acid & \\
\hline 9 & n-Nonadecanol-1 & Fatty alcohol & \\
\hline 10 & 9-octadecenoic acid & Oleic acid & \\
\hline 11 & Octadecanal & Fatty Aldehyde & $\ldots$ \\
\hline 12 & Ocadecanoic acid & Stearic acid & \\
\hline 13 & $\gamma$-Tocopherol & Vitamin E compound & 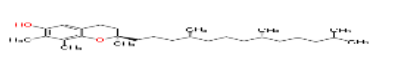 \\
\hline 14 & Ergost-5-en-3-ol, (3.beta.,24r)- & Campesterol & \\
\hline 15 & Stigmasta-5,22-dien-3-ol & Stigmasterol & \\
\hline 16 & Stigmast-5-en-3-ol, (3.beta.)- & $\beta$-Sitosterol & \\
\hline 17 & $\begin{array}{l}\text { 2,3-Dihydro-3,5-dihydroxy-6-methyl- } \\
\text { 4h-pyran }\end{array}$ & Pyranone & \\
\hline 18 & 2-Methoxy-4-vinylphenol & Phenolic compound & \\
\hline
\end{tabular}


Sunita Arora et al. Int. Res. J. Pharm. 2017, 8 (6)

\begin{tabular}{|l|l|l|l|}
\hline 19 & Tetradecanoic acid & Myristic acid & \\
\hline 20 & Hexadecanoic acid, methyl ester & Fatty acid methyl ester & \\
\hline 21 & $\begin{array}{l}9,12-\text { Octadecadienoic acid } \quad(\mathrm{z}, \mathrm{z},)-, \\
\text { methyl ester }\end{array}$ & Fatty acid methyl ester & \\
\hline 22 & $\begin{array}{l}9,12,15-\text { Octadecatrienoic acid, methyl } \\
\text { ester, (z,z,z)- }\end{array}$ & Fatty acid methyl ester & \\
\hline
\end{tabular}

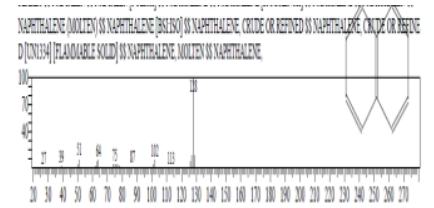

Figure 4: Mass spectrum of Naphthalene

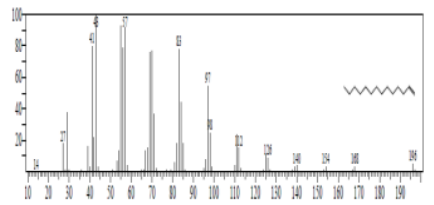

Figure 7: Mass spectrum of 1-Tetradecene

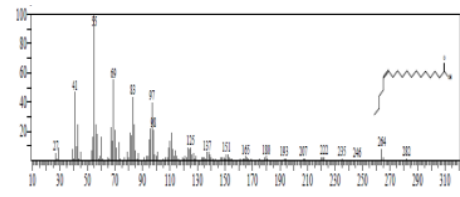

Figure 10: Mass spectrum of cis-13Octadecenoic acid

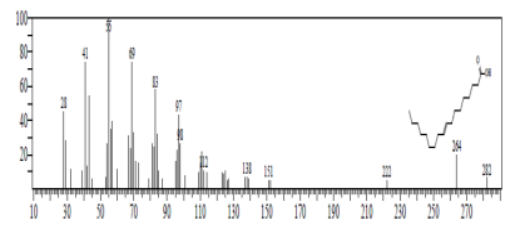

Figure 13: Mass spectrum of 9-octadecenoic acid

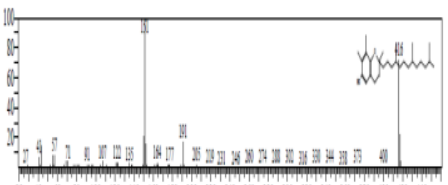

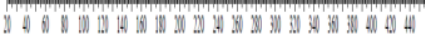

Figure 16: Mass spectrum of $\boldsymbol{\gamma}$-Tocopherol

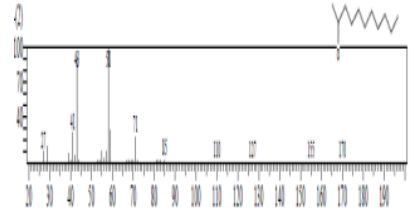

Figure 5: Mass spectrum of 2-Undecanone

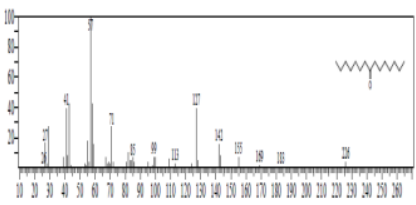

Figure 8: Mass spectrum of 8-Pentadecanone

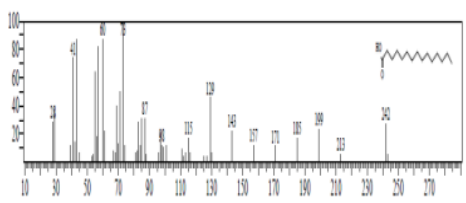

Figure 11: Mass spectrum of Pentadecanoic acid

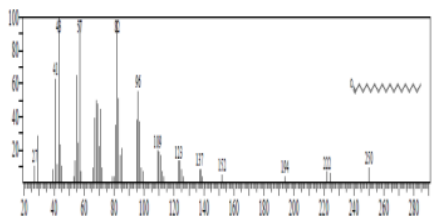

Figure 14: Mass spectrum of Octadecanal

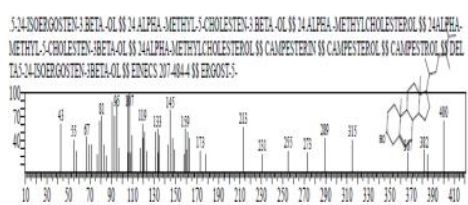

Figure 17: Mass spectrum of ERGOST-5-EN3-OL, (3. BETA ., 24R)-

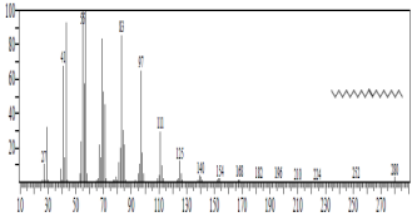

Figure 6: Mass spectrum of 9-Eicosene, (E)-

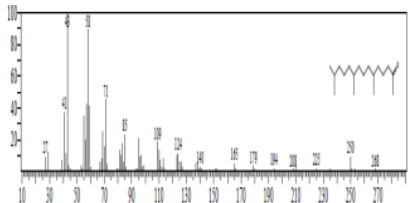

Figure 9: Mass spectrum of 2-Pentadecanone, $6,10,14$-trimethyl-

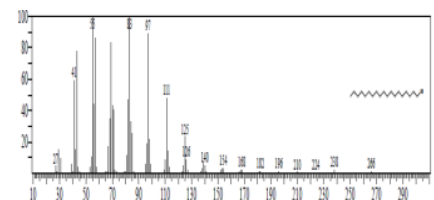

Figure 12: Mass spectrum of n-Nonadecanol-

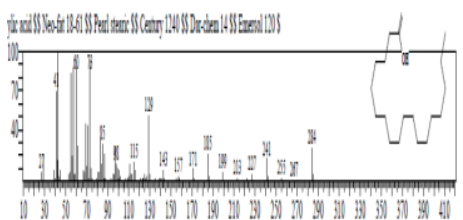

Figure 15: Mass spectrum of Ocadecanoic acid

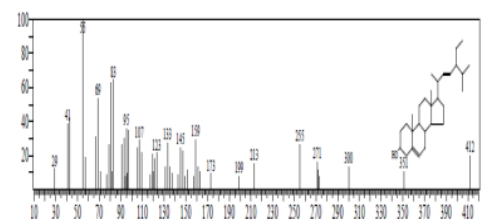

Figure 18: Mass spectrum of Stigmasta-5, 22dien-3-ol 


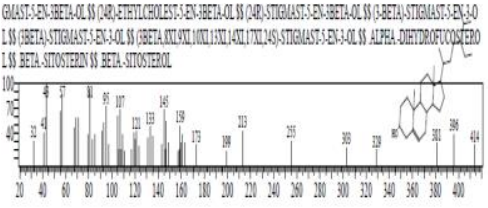

Figure 19: Mass spectrum of Stigmast-5-en-3ol, (3.beta.)-

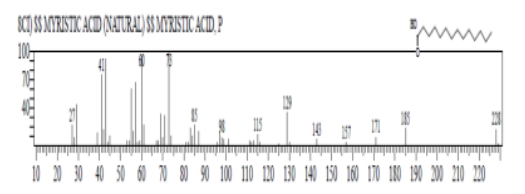

Figure 22: Mass spectrum of Tetradecanoic acid

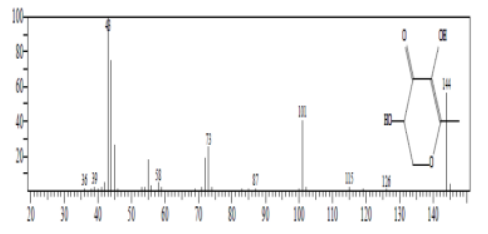

Figure 20: Mass spectrum of 2, 3-Dihydro-3, 5-dihydroxy-6-methyl-4h-pyran

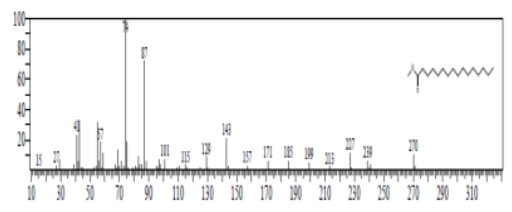

Figure 23: Mass spectrum of Hexadecanoic acid, methyl ester

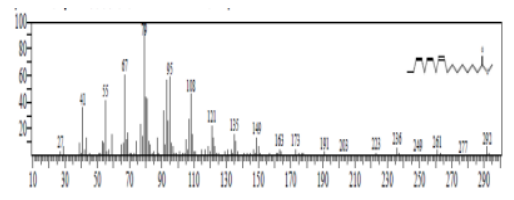

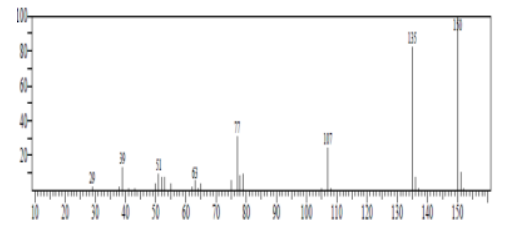

Figure 21: Mass spectrum of 2-Methoxy-4vinylphenol

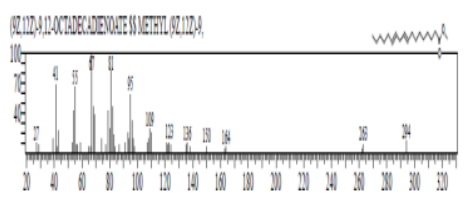

Figure 24: Mass spectrum of 9, 12Octadecadienoic acid (z, z,)-, methyl ester

Figure 25: Mass spectrum of 9, 12, 15-Octadecatrienoic acid, methyl ester, (z, z, z)-

\section{CONCLUSION}

22 bioactive compounds were identified in methanol extract of whole plant of $C$. ciliaris by GC-MS analysis. The results revealed presence of higher no. of compounds in methanol extract of leaf followed by root and stem. GC-MS study has exploited the potential of $C$. ciliaris. This plant can be a good source of phytoconstituents like alkane, carboxylic acid, phytosterol, aldehyde, ketone, fatty acid ester, fatty alcohol, terpenes and Vitamin E. Ergost-5-en-3-ol, (3.beta., 24r)(Campesterol) and Stigmasta-5, 22-dien-3-ol (Stigmasterol) both are anti-cancerous phytosterols. Stigmast-5-en-3 $\beta$-ol $(\beta$ Sitosterol), a phytosterol shows anti-inflammatory, anti-pyretic, anti-arthritic, anti-ulcer, insulin releasing and estrogenic effects. In future, the isolation and purification of above mentioned compounds analyzed from various parts of this plant would be fruitful for the pharmaceutical companies to formulate novel drugs and herbal medications for treating various ailments. It could be concluded that $C$. ciliaris L. contains numerous bioactive compounds. So it's recommended as a plant of pharmaceutical importance. However further studies are needed to undertake its bioactivity and toxicity profile.

\section{ACKNOWLEDGEMENTS}

I am grateful to the Department of Botany, Jai Narain Vyas, University, Jodhpur \& AIRF (Advanced Instrumentation Research Facility), Jawaharlal Nehru University, New Delhi, for assisting laboratory \& Instrumentation facilities.

\section{REFERENCES}

1. Kalaisezhiyen P, Vadivukkarasi SK. GC-MS evaluation of Chemical constituents from methanolic leaf extract of Kedrostis foetidissima (Jacq.) Cogn. Asian Journal of Pharmaceutical and Clinical Research 2012; 5 Suppl 4:7781.

2. Vaidya ADB, Devasagayam TPA. Current Status of Herbal Drugs in India: An overview. Journal of Clinical Biochemistry and Nutrition 2007; 41(1):1-11.
3. Raskin I. et al. Plants and human health in the twenty-first century. Trends in Biotechnology 2002; 20(12): 522-531.

4. Karimi E, Jaafar HZE. HPLC and GC-MS determination of bioactive compounds in microwave obtained extracts of three varieties of Labisia pumila benth. Molecules 2011; 16(8): 6791- 6805.

5. Vuorelaa P. et al. Natural products in the process of finding new drug candidates. Current Medicinal Chemistry 2004; 11(11):1375- 1389.

6. Bai S, Seasotiya L, Malik A, Bharti P, Dalal S. GC-MS analysis of chloroform extract of Acacia nilotica L. leaves. Journal of Pharmacognosy and Phytochemistry 2014; 2(6): 79-82.

7. Singariya $\mathrm{P}$, Kumar $\mathrm{P}$, Mourya, KK. Isolation of new steroids of Kala Dhaman grass (Cenchrus setigerus) and evaluation of their bioactivity. Brazilian Archives of Biology and technology 2014; 57(1): 62-69.

8. Harborne JB. Methods of plant analysis. In: Harborne JB, editor. Phytochemical Methods. 2nd ed. Chapman and Hall, London; 1984. p.5-6.

9. Nguyen HP, Kimaru IW. GC-MS Analysis of an Herbal Medicinal Remedy to Identify Potential Toxic Compounds. Advances in Pharmaceutical Analysis 2014; 12(4):14-18.

10. Bradford PG, Awad AB. Phytosterols as anticancer compounds. Molecular Nutrition \& Food Research 2007; 51(2):161-170.

11. Zawistowski J. Tangible health benefits of phytosterol functional foods. In: J. Smith, E. Charter editors. Functional Food Product Development. Oxford, UK: Wiley Blackwell; 2010.p.362-387.

12. Patra A, Jha S, Murthy PN, Manik, Sharone A. Isolation and characterization of stigmast- 5-en-3 $\beta$-ol ( $\beta$-sitosterol) from the leaves of Hygrophila spinosa T. Anders. International Journal of Pharma Sciences and Research 2010; 1(2):95100.

13. Seo S. et al. Structure-activity relationship of aliphatic compounds for nematicidal activity against pine wood nematode (Bursaphelenchus xylophilus). Journal of Agricultural and Food Chemistry 2010; 58(3):1823-27. 
14. Markkas N, Govindharajalu M. Determination of phytocomponents in the methanolic extract of Mollugo cerviana by GC-MS analysis. International Journal of Research in Biological Sciences 2015; 5(4):26-29.

15. Vijisaral ED, Subramanian A. GC-MS Analysis of Ethanol Extract of Cyperus rotundus Leaves. International Journal of Current Biotechnology 2014; 2(1):19-23.

16. Akpuaka A, Ekwenchi MM, Dashak DA, Dildar A. Biological Activities of Characterized Isolates of $n$-Hexane Extract of Azadirachta indica A.Juss (Neem) Leaves. New York Science Journal 2013; 6(6):119-124.

17. Abdelhamid MS, Kondratenko EI, Lomteva NA. GC-MS analysis of phytocomponents in the ethanolic extract of
Nelumbo nucifera seeds from Russia. Journal of Applied Pharmaceutical Science 2015; 5 (4):115-118.

18. Sermakkani M, Thangapandian V. GC-MS analysis of Cassia italica leaf methanol extract. Asian Journal of Pharmceutical and Clinical Research 2012; 5(2): 90-94.

\section{Cite this article as:}

Sunita Arora et al. Screening and evaluation of bioactive components of Cenchrus ciliaris L. by GC-MS analysis. Int. Res. J. Pharm. 2017;8(6):69-76 http://dx.doi.org/10.7897/22308407.08699

\section{Source of support: Nil, Conflict of interest: None Declared}

Disclaimer: IRJP is solely owned by Moksha Publishing House - A non-profit publishing house, dedicated to publish quality research, while every effort has been taken to verify the accuracy of the content published in our Journal. IRJP cannot accept any res ponsibility or liability for the site content and articles published. The views expressed in articles by our contributing authors are not necessarily thos e of IRJP editor or editorial board members. 